\title{
Producción de etanol a partir de cebada no malteada hidrolizada con $\alpha$ y $\beta$ amilasas comerciales
}

\author{
Carolina Espitia-Rocha $^{1}$, Ivonne Gutierrez-Rojas ${ }^{2 *}$, Helberth Espitia-Rivera ${ }^{1}$ \\ ${ }^{1}$ Destilería Premier LTDA. Centro Industrial Chapeton (Vía Nevado), Ibagué, Colombia. \\ ${ }^{2}$ Grupo de Biotecnología Ambiental e Industrial (GBAI), Departamento de Microbiología, Facultad de Ciencias, \\ Pontificia Universidad Javeriana, Bogotá, D.C., Colombia. \\ *ivonne.gutierrez@javeriana.edu.co
}

Recibido: 22-10-2009; Aceptado: 19-11-2009

\begin{abstract}
Resumen
Objetivo. Establecer la concentración óptima de $\alpha$ y $\beta$ amilasas comerciales para la obtención de etanol a partir de cebada sin maltear. Materiales y métodos. Cebada no malteada fue hidrolizada con concentraciones variables de $\alpha$ y $\beta$ amilasas comerciales (Genencor Internacional), bajo las condiciones establecidas por el fabricante. Los productos de hidrólisis fueron utilizados como sustrato para la producción de etanol con Sacharomyces cerevisiae. Adicionalmente se realizó un ensayo referencia con cebada malteada, bajo las condiciones establecidas por la destilería. Resultados. Se obtuvo un porcentaje de hidrólisis del almidón de $89,4 \%$ cuando se adicionaron de $\alpha$ y $\beta$ amilasas a una concentración de $1 \mathrm{gL}^{-1}$, adicionalmente se obtuvo la máxima producción de etanol (5,02\%), siendo significativamente más alta que cuando se utilizó cebada malteada $(3,76 \%)$. Conclusiones. Se demostró que se puede producir etanol a partir de almidón de cebada sin maltear empleando $\alpha$ y $\beta$-amilasas comerciales, aunque es necesario optimizar el proceso ya que es más costoso comparado con el tradicional, utilizando cebada malteada.
\end{abstract}

Palabras clave: fermentación, hidrólisis del almidón, Saccharomyces cerevisiae, $\alpha$-amilasas, $\beta$-amilasas.

\begin{abstract}
Ethanol production from hydrolyzed unmalted barley using commercial $\alpha$ - and $\beta$-amylase. Objective. To find the optimum concentration of commercial $\alpha$ - and $\beta$-amylase for the obtainment of ethanol from unmalted barley. Materials and methods. Unmalted barley was hydrolyzed using various concentrations of commercial $\alpha$ - and $\beta$-amylase (Genencor International), following conditions established by the manufacturer. The products of hydrolysis were used as substrates for the production of ethanol by Saccharomyces cerevisiae. In addition, a reference assay was performed using malted barley following the conditions established by the distillery. Results. The percentage of starch hydrolysis was $89.4 \%$ when adding $\alpha$-and $\beta$-amylase at a concentration of $1 \mathrm{~g} \mathrm{~L}^{-1}$. Moreover, this concentration of amylases yielded a maximum ethanol production (5.02\%) significantly higher than when malted barley was used (3.76 $\%$ ). Conclusions. It was demonstrated that ethanol can be obtained from starch of unmalted barley by adding commercial $\alpha$ - and $\beta$ amylase. However, optimization of the process is required due to the higher costs when compared to the traditional process with malted barley.
\end{abstract}

Key words: $\alpha$-amylase, $\beta$-amylase, fermentation, Saccharomyces cerevisiae, starch hydrolysis. 


\section{Resumo}

Produção de etanol a partir de cevada não maltada hidrolisada com $\alpha$ e $\beta$ amilases comerciais. Objetivo. Estabelecer a concentração ideal de $\alpha$ e $\beta$ amilases comerciais para a obtenção de etanol a partir de cevada não malteada. Materiais e métodos. Cevada não malteada foi hidrolisada com diferentes concentrações de $\alpha$ e $\beta$ amilases comerciais (Genencor Internacional), sob as condições estabelecidas pelo fabricante. Os produtos da hidrólise foram utilizados como substrato para produção de etanol com Saccharomyces cerevisiae. Além disso, se realizou um teste de referência com cevada malteada, sob as condições estabelecidas pela destilaria. Resultados. Se obtive uma percentagem de hidrólise do amido de $89,4 \%$ ao adicionar á e â amilases na concentração de $1 \mathrm{gL}^{-1}$, adicionalmente se obtive a produção máxima de etanol (5,02\%), sendo significativamente maior do que quando é usada cevada malteada (3,76\%). Conclusões. Foi demonstrado que o etanol pode ser produzido a partir de amido de cevada não maltada com $\alpha$ e $\beta$-amilases comerciais, embora seja necessário aperfeiçoar o processo, porque é mais caro, em comparação com o tradicional, utilizando cevada maltada.

Palavras-chave: fermentação, hidrólise do amido, Saccharomyces cerevisiae, $\alpha$-amilase, $\beta$-amilases.

\section{Introducción}

Tradicionalmente en el proceso de elaboración de whisky o de cerveza se requiere de la cebada como materia prima esencial, la cual debe ser previamente sometida a un proceso de malteado. Durante el malteado, el grano crudo de cebada germina de manera controlada y el almidón de la cebada es hidrolizado produciendo azúcares menos complejos, los cuales pueden ser utilizados por Saccharomyces cerivisiae para la producción de etanol. Algunas de las enzimas necesarias para convertir las reservas de la semilla de cebada en los componentes de la malta están presentes en el grano maduro, mientras que las otras son sintetizadas en el embrión durante la germinación $(1,2)$.

Uno de los mayores inconvenientes a los que se enfrenta la industria licorera Colombiana es el bajo número de malterías que produzcan y comercialicen cebada malteada con los requerimientos de calidad que exige la industria (3), lo que hace necesaria la importación de cebada transformada; generando de esta forma costos mayores para las empresas que requieren de la materia prima en estas condiciones. Como alternativa se encuentra el uso de enzimas comerciales producidas por microorganismos, que hidrolicen el almidón de la misma manera que lo hacen las enzimas del grano de cebada. Este proceso ha sido ampliamente utilizado en varias industrias de alimentos y bebidas, especialmente en la obtención de jarabes fructosados y etanol $(4,5)$. Por lo general se utilizan $\alpha$-amilasa (E.C.3.2.1.1), $\beta$-amilasa (E.C. 3.2.1.2) y glucoamilasa (E.C.3.2.1.3), obtenidas de microorganismos como Bacillus licheniformis y Aspergillus niger (6). Algunas de las ventajas que presenta la hidrólisis enzimática del almidón son, alta tasa de reacción, alta estabilidad de las enzimas y disminución de la viscosidad del medio de reacción. Por otro lado, los parámetros básicos que afectan este proceso son el pH del medio, la concentración del sustrato, la concentración de la enzima y la temperatura. Los valores óptimos de estos parámetros varían en función de la fuente de ob- tención de la enzima (7). Frecuentemente, se utilizan $\alpha$ amilasas termoestables, activas en un rango de temperatura entre 90 y $110 \pm 1^{\circ} \mathrm{C}$, con una concentración de sustrato entre 20 y $35 \%$ (p/v), un rango de $\mathrm{pH}$ entre 6 y $8 \pm 0,2$ y una concentración de enzima entre 0,03 y $1 \%$ (v/v) $(5,7)$.

El objetivo de este trabajo fue establecer la concentración óptima de $\alpha$ y $\beta$ amilasas comerciales para la obtención de etanol a partir de cebada sin maltear, como alternativa al uso de cebada malteada importada.

\section{Materiales y métodos}

El presente trabajo se realizó en el laboratorio de control de calidad de la Destilería Premier LTDA, con el respaldo técnico del laboratorio de Bromatología de la Secretaría de Salud del Tolima, en la ciudad de Ibagué (Colombia).

\section{Materia prima}

Los ensayos se realizaron empleando cebada no malteada cultivada en la región Cundiboyacense, Colombia (Concentración de almidón, $60 \%$ (p/p)) y cebada malteada importada de Malterías Unidas, Chile (Concentración de almidón, $80 \%(\mathrm{p} / \mathrm{p}))$.

\section{Enzimas y microorganismo}

Se utilizaron las enzimas comerciales Multifect ${ }^{\circledR}$ AA 21L y Multifect ${ }^{\circledR}$ AA 23L de Genencor Internacional, Inc. De acuerdo con la información dada por la casa comercial, la primera es una $\alpha$-amilasa estable a altas temperaturas (105 a $\left.110 \pm 1^{\circ} \mathrm{C}\right)$ y valores bajos de $\mathrm{pH}(5,0-5,8 \pm 0,2)$, tiene una actividad de $17400 \mathrm{ULg}^{-1}$ (mínimo), Unidad de Licuefacción (UL) definida como el tiempo de digestión requerido para producir cambio de color en una solución de yodo, indicando un estado definido de dextrinización del almidón, bajo condiciones de ensayo definidas. La segunda enzima, es una $\beta$-amilasa efectiva en un rango amplio 
de $\mathrm{pH}(4,5-7,5 \pm 0,2)$, con un óptimo de 5,5 y en un rango de temperatura de 50 a $65 \pm 1^{\circ} \mathrm{C}$, con una temperatura óptima de $60^{\circ} \mathrm{C}$, tiene una actividad de $1230{ }^{\circ} \mathrm{PDg}^{-1}$ (mínimo), Grado de Poder Diastásico ( $\left.{ }^{\circ} \mathrm{PD}\right)$ definido como la cantidad de enzima contenida en $0,1 \mathrm{~mL}$ de una solución al $5 \%(\mathrm{v} / \mathrm{v})$, que puede producir cantidad suficiente de azúcares reductores para reducir $5 \mathrm{~mL}$ de una solución de Fehling, cuando es incubada con $100 \mathrm{~mL}$ del sustrato por $1 \mathrm{~h}$ a $20 \pm 1^{\circ} \mathrm{C}$.

La levadura utilizada para la fermentación alcohólica fue S. cerevisiae Safwhisky M-1, de la casa comercial Fermentis, en una presentación de levadura seca instantánea.

\section{Hidrólisis del almidón}

Todos los ensayos de hidrólisis se llevaron a cabo en erlenmeyer de $2 \mathrm{~L}$ en agitador orbital termostatado, a las mismas condiciones de experimentación. Se tomaron 250 $\mathrm{g}$, de cebada sin maltear y se mezclaron con $500 \mathrm{~mL}$ de agua destilada a $22^{\circ} \mathrm{C}$ durante 15 minutos, seguidamente se adicionaron $500 \mathrm{~mL}$ de tampón acetato de sodio $\left(\mathrm{CH}_{3} \mathrm{COONa}\right)$ 0,05M (pH 5,5 $\left.\pm 0,2\right)(8,9)$. La hidrólisis del almidón se realizó en dos etapas. En la primera, licuefacción, se añadió la concentración de $\alpha$-amilasa a evaluar de acuerdo al diseño experimental mostrado en la tabla 1 , estas concentraciones fueron calculadas tomando como base la concentración recomendada por la casa comercial. Se incubó durante 2 horas a $95 \pm 1^{\circ} \mathrm{C}$. Para la sacarificación, se adicionó $\beta$-amilasa (Tabla 1) y se incubó durante 6 horas a $57 \pm 1^{\circ} \mathrm{C}(10)$. La hidrólisis de la mezcla se detuvo por adición de $\mathrm{HCl} 2 \mathrm{M}$ hasta $\mathrm{pH}$ 1,5-1,6 $\pm 0,2$. Finalmente se ajustó el pH a $5-6 \pm 0,2$ (11). Al final de la hidrólisis se determinaron azúcares reductores (12). Todos los ensayos se realizaron por triplicado.

Como referencia se realizó un ensayo con cebada malteada. Para tal efecto, se tomaron $250 \mathrm{~g}$, de cebada malteada y se adicionaron a 1 litro de agua destilada a $45 \pm 1^{\circ} \mathrm{C}$ durante 55 minutos, posteriormente se incubó bajo las condiciones estandarizadas en la destilería, con el fin de asegurar que los almidones de la cebada se transformen en azúcares, de la siguiente manera: $52 \pm 1^{\circ} \mathrm{C}$ por $30 \mathrm{~min}, 62 \pm 1^{\circ} \mathrm{C}$ por 1 hora y $72 \pm 1^{\circ} \mathrm{C}$ por $30 \mathrm{~min}$, al cabo de este tiempo se separó el mosto de cebada del resto de los afrechos de la misma, obteniéndose así un mosto para el proceso de fermentación, al cual se le determinó azúcares reductores (12). El control negativo se desarrolló bajo las mismas condiciones amilolíticas y fermentativas del ensayo de referencia, utilizando cebada sin maltear y sin adición de enzimas durante el proceso hidrolítico. Todos los ensayos se hicieron por triplicado.

El rendimiento de la hidrólisis enzimática fue calculado de acuerdo a la ecuación reportada por Chen y col (13):

$$
R H(\%)=\frac{A R * 0,9 * 100}{P}
$$

Donde: $R H=$ Rendimiento de hidrólisis

$$
\begin{aligned}
& A R=\text { Concentración de azúcares reductores } \\
& P=\text { Concentración de polisacárido en el sustrato }
\end{aligned}
$$

Tabla 1. Resumen del diseño experimental

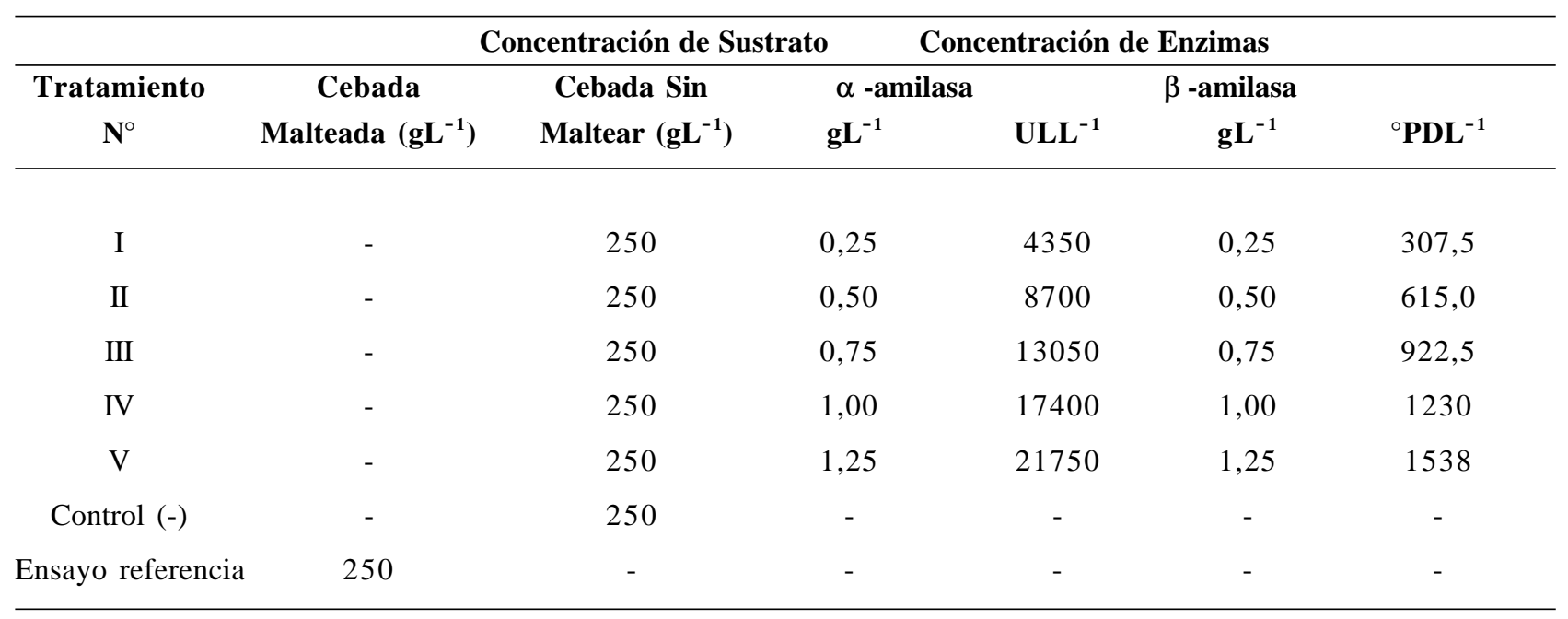




\section{Fermentaciones}

Para la preparación del inóculo, $1 \mathrm{~g}$ de $S$. cerevisiae se inoculó en 1 litro de mosto preparado bajo las condiciones especificadas para el ensayo referencia, sin complementos nutricionales o ajuste de $\mathrm{pH}$. Se utilizó un erlenmeyer de $2 \mathrm{~L}$ y se incubó en agitación, 100 rpm, a 30 $\pm 1^{\circ} \mathrm{C}$, por $24 \mathrm{~h}(14)$.

Las fermentaciones se llevaron a cabo en erlenmeyer de $2 \mathrm{~L}$ con un volumen de trabajo de 1L. Para esto, se inocularon $100 \mathrm{~mL}$ de un cultivo obtenido como se indicó anteriormente (porcentaje de inóculo, 10\% (v/v)), con una concentración de $1 \times 10^{6}$ células $\mathrm{mL}^{-1}$, en $900 \mathrm{~mL}$ del producto del proceso hidrolítico (12), de acuerdo al diseño experimental presentado en la tabla 1 . Se adicionaron $1,5 \mathrm{gL}^{-1} \mathrm{de}$ sulfato de amonio como fuente de nitrógeno. Posteriormente, se incubó en aerobiosis a $35 \pm 1^{\circ} \mathrm{C}, 100 \mathrm{rpm}$, por 36 horas, pasado este tiempo se cambió el tapón de algodón por un tapón de caucho con filtro de salida de $\mathrm{CO}_{2}$, para dar inicio a la fase de fermentación en condiciones anaerobias y se incubó hasta completar 84 horas. Se tomaron muestras cada 12 horas para determinar biomasa. La concentración de azúcares reductores y de etanol se determinó al final del proceso de fermentación. Todos los ensayos se realizaron por triplicado.

\section{Técnicas analíticas}

La concentración de la biomasa en células $\mathrm{mL}^{-1}$ se cuantificó por conteo de células en cámara de Neubauer (15). La concentración de almidón se determinó por la técnica de yodo - yoduro y la de azúcares reductores por medio del método de Fehling (16). Para la determinación de la concentración de etanol se utilizó el método por destilación y estimación del contenido alcohólico por determinación de la gravedad específica. En este método el producto se somete a destilación en condiciones específicas y se determina la densidad del destilado, con este valor y utilizando tablas correspondientes se calcula el grado alcoholimétrico (17).

\section{Análisis estadístico}

Se realizó un análisis de varianza y se aplicó la prueba de Tukey para determinar sí entre los niveles de cada factor hay diferencias significativas, en el programa estadístico SPSS versión 17.0. Se trabajó con un nivel de significancia del 95\%. Las variables de respuesta fueron biomasa ( $\log$ células $\mathrm{mL}^{-1}$ ), concentración de azúcares reductores (\%) y producción de etanol (\%).

\section{Resultados y discusión}

En la figura 1, se muestra el efecto de la concentración de enzimas ( $\alpha$ y $\beta$ amilasas) sobre la hidrólisis del almidón de cebada no malteada. Se observa que la concentración de azúcares reductores se incrementa en el medio a medida que aumenta la concentración de enzimas utilizada para la hidrólisis, hasta alcanzar un valor máximo $(59,6 \%)$ cuando se utiliza una concentración de enzimas de $1 \mathrm{gL}^{-1}$, como es de esperarse a esta concentración se alcanza el mayor porcentaje de hidrólisis $(89,4 \%)$. Sin embargo, cuando se utiliza una concentración de enzimas mayor, $1,25 \mathrm{gL}^{-1}$, tanto la concentración de azúcares reductores $(43,5 \%)$, como el rendimiento de hidrólisis $(65,3 \%)$ disminuyen. Estos resultados concuerdan con lo reportado en estudios similares, Govindasamy y col, observaron una relación lineal entre el incremento de la concentración de enzimas (de 0 a 75\%) y el aumento de la sacarificación, sin embargo, incrementos superiores al $75 \%$ no mostraron aumento en la sacarificación, lo cual responde a la saturación de la enzima (6). Kolusheva y Marinova observaron este efecto para incrementos superiores al $85 \%$ (7) y en el presente estudio para incrementos superiores al $80 \%$.

Cuando se comparan los resultados obtenidos en estos ensayos con el control negativo (cebada no malteada, sin adición de enzimas), se observa que en todos los casos la adición de enzimas amilolíticas aumenta significativamente $(\mathrm{P}<0,05)$ la cantidad de azúcares reductores liberados al medio de cultivo (control negativo: $2,57 \%$ ) y el rendimiento de hidrólisis (control negativo: 0\%). Este comportamiento corresponde al esperado, ya que para que se hidrolice el almidón presente en el grano de cebada, es necesario que este se someta a un proceso de maltaje, en el cual la hidrólisis del almidón es realizada por la acción concertada de 4 enzimas presentes en éste, $\alpha$ y $\beta$ amilasas y cantidades pequeñas de $\alpha$ glucosidasa y dextrinasa, cuya acción combinada es conocida como poder diastásico (DP). La $\alpha$-amilasa es sintetizada durante la germinación del grano, mientras que la $\beta$-amilasa es sintetizada durante el desarrollo del mismo y se encuentra acumulada en éste en un 1 a $2 \%$ del nitrógeno total en el grano maduro $(18,19)$. Este comportamiento es evidente en el ensayo de referencia (cebada malteada, sin adición de enzimas), en donde se obtiene un porcentaje de azúcares reductores del $37 \%$ y un rendimiento de hidrólisis del $41 \%$, sin necesidad de la adición de enzimas exógenas, estos resultados no son estadísticamente diferentes $(\mathrm{P}<0,05)$ de los obtenidos para los ensayos con adición de enzimas correspondientes a las concentraciones de 0,$25 ; 0,5 ; 0,75$ y $1,25 \mathrm{gL}^{-1}$, pero son significativamente menores a los obtenidos con una concentración de $1 \mathrm{gL}^{-1}$. 


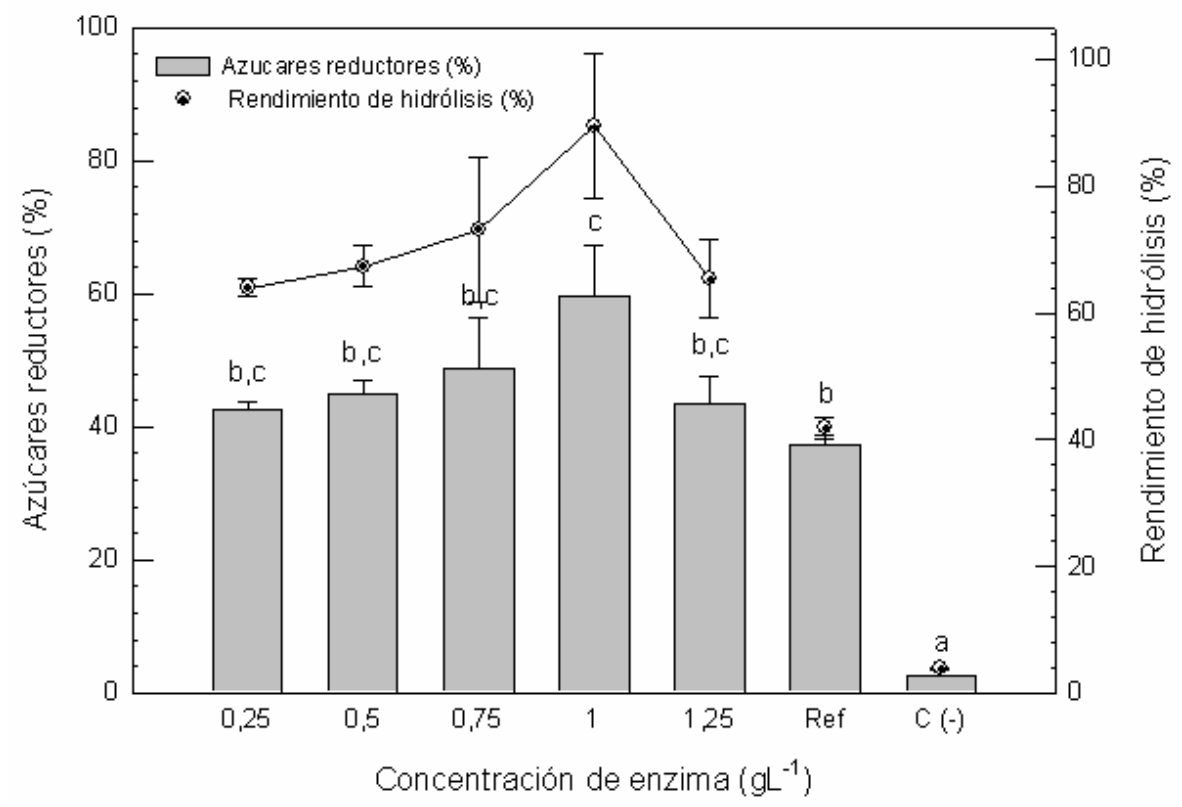

Figura 1. Efecto de la concentración de enzimas sobre la liberación de azúcares reductores y rendimiento de hidrólisis del almidón de cebada no malteada. Las letras corresponden a los Grupos de Tukey $(\mathrm{P}<0,05)$.

Cuando los productos de hidrólisis se utilizaron como sustrato para el crecimiento de Sacharomyces cerevisiae, se obtuvo el comportamiento cinético que se muestra en la figura 2. Para todos los casos se observa que el microorganismo no presenta fase de adaptación, iniciando el crecimiento en fase exponencial desde la hora 0 hasta la hora 36 , en donde se evidencia una desaceleración de la velocidad de crecimiento hasta la hora 48 , tiempo en el que se obtiene la máxima concentración de células en todos los tratamientos. Este comportamiento corresponde al esperado, ya que en esta fase ( $0 \mathrm{~h}$ a $36 \mathrm{~h}$ ) el cultivo se encuentra en condiciones aeróbicas, y por lo tanto el metabolismo es mayoritariamente oxidativo, los azúcares son rápidamente oxidados a $\mathrm{CO}_{2}$ y agua liberando una gran cantidad de energía utilizada para la formación de biomasa. Adicionalmente, en esta etapa se da la síntesis de lípidos (esteroles y ácidos grasos insaturados), los cuales son esenciales en la integridad de la membrana y la tolerancia a etanol en etapas posteriores $(20,21)$. En la hora 36, las condiciones de fermentación varían presentándose limitación por oxígeno, lo que coincide con la desaceleración de la velocidad de crecimiento y el inicio de la fase estacionaria, debido probablemente al cambio en el metabolismo celular de oxidativo a fermentativo, en este último se presenta un rendimiento de sustrato en biomasa mucho más bajo que el obtenido por vía oxidativa, por lo tanto se observa un menor crecimiento y un mayor incremento en la concentración de etanol $(21,22)$. En el caso del control nega- tivo, el descenso en la población celular a partir de la hora 48 es mucho más marcado, debido a que en éste la concentración inicial de azúcares reductores $(2,57 \%)$ es mucho menor y es probable que a esta etapa del cultivo ya se hayan agotado, hecho que también se verá reflejado en la producción de etanol, como se mostrará más adelante. En la figura 3, se muestra la productividad en biomasa obtenida para cada uno de los tratamientos, como es de esperarse las más bajas corresponden a los tratamientos con menores concentraciones iniciales de azúcares reductores (control negativo, 2,57 \% y tratamiento I, 42,6 \%). Por otro lado, las obtenidas en los demás tratamientos no muestran gran diferencia con el ensayo de referencia, lo que demuestra que las concentraciones de azúcares reductores obtenidas en los hidrolizados enzimáticos de cebada no malteada fueron las necesarias para el desarrollo de $S$. cerevisiae, lográndose un comportamiento muy cercano al obtenido tradicionalmente en la destilería (ensayo de referencia con cebada malteada).

En cuanto a la producción de etanol (Figura 4), se observa una tendencia idéntica a la mostrada en los resultados de hidrólisis (Figura 1), es decir que a mayor concentración de enzimas utilizada en la hidrólisis mayor concentración de etanol producida, hasta un máximo de 5,05\% para el tratamiento en donde se utiliza $1 \mathrm{gL}^{-1}$ de enzimas. Este comportamiento es de esperarse, debido a que la disponibilidad de azúcares reductores en el medio después de la fase 


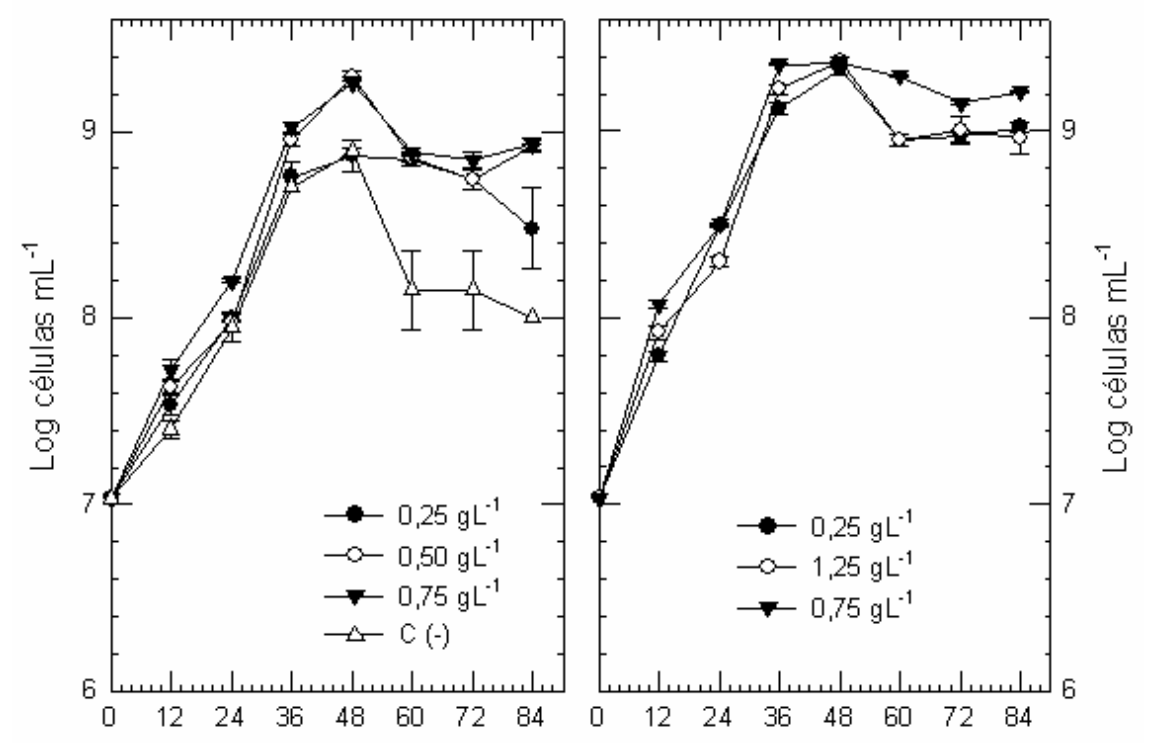

Tiempo (horas)

Figura 2. Cinética de crecimiento de Sacharomyces cerevisiae en los productos de hidrólisis obtenidos a partir de diferentes concentraciones de enzimas.

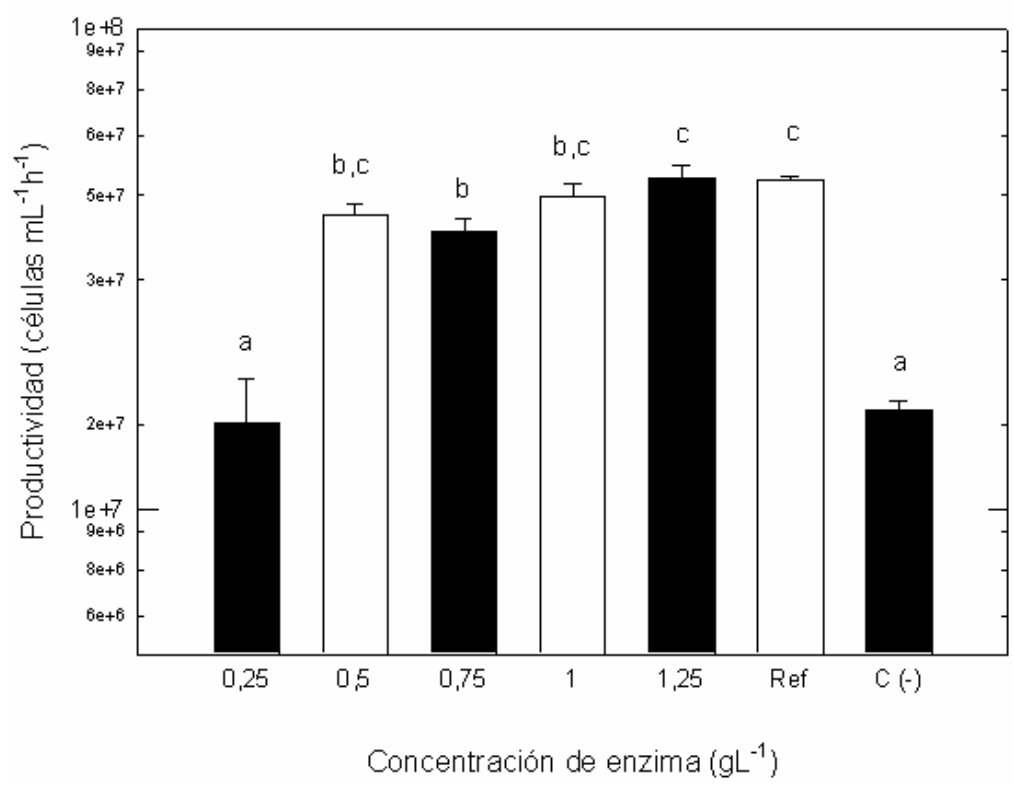

Figura 3. Productividad máxima (células.mL.h ${ }^{-1}$ ) de Sacharomyces cerevisiae en los productos de hidrólisis obtenidos a partir de diferentes concentraciones de enzimas. Las letras corresponden a los Grupos de Tukey $(\mathrm{P}<0,05)$. 


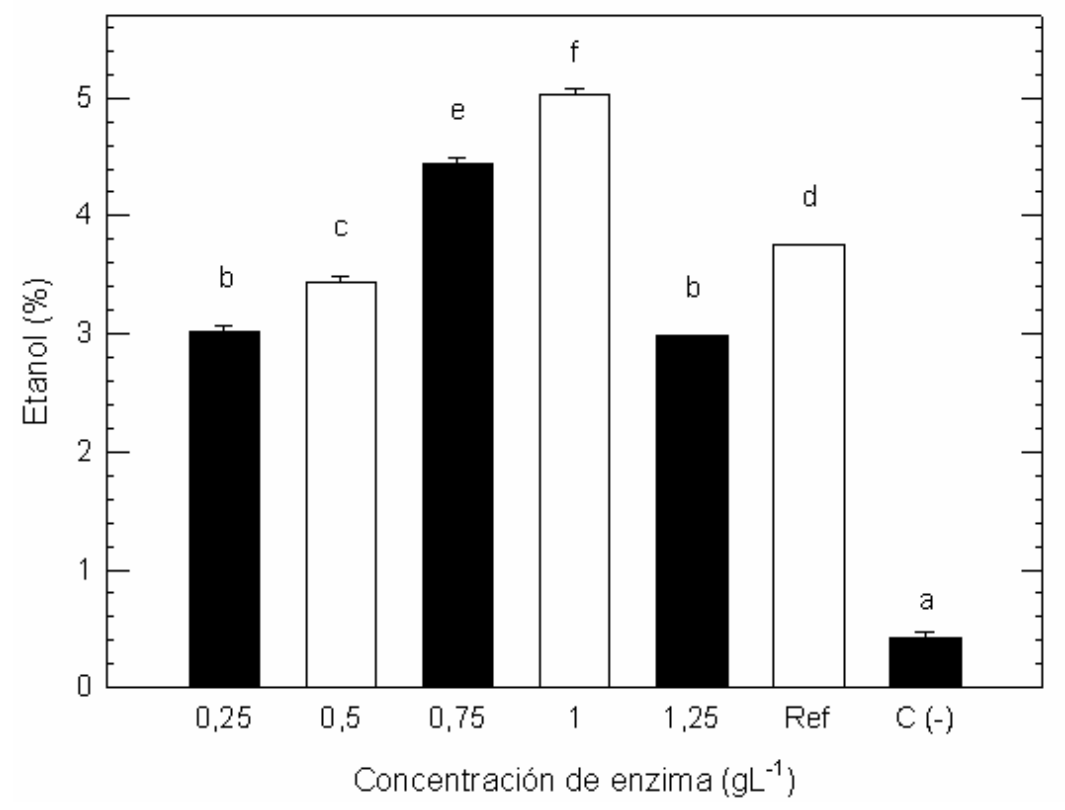

Figura 4. Producción de etanol en los productos de hidrólisis obtenidos a partir de diferentes concentraciones de enzimas. Las letras corresponden a los Grupos de Tukey $(\mathrm{P}<0,05)$.

de crecimiento exponencial en condiciones aerobias, es mayor en los tratamientos en donde se inicia con concentraciones mayores, hecho que es muy evidente en el control negativo, en donde se obtiene una producción de etanol muy baja $(0,43 \%)$. Cuando se compara la producción de etanol en los hidrolizados enzimáticos con la obtenida en el ensayo de referencia (cebada malteada: 3,76\%), se observa que en los tratamientos en donde se usaron concentraciones de enzimas de $0,75 \mathrm{gL}^{-1}$ y $1 \mathrm{gL}^{-1}$, se logran concentraciones significativamente más altas $(\mathrm{P}<0,05)$ de etanol $\left(0,75 \mathrm{gL}^{-1}, 4,44 \% ; 1 \mathrm{gL}^{-1}, 5,02 \%\right)$. Adicionalmente, los destilados obtenidos en los diferentes tratamientos se sometieron a una prueba sensorial (resultados no mostrados, realizados por el panel de evaluación sensorial de la Destilería Premier), con el fin de determinar una posible alteración en la calidad organoléptica del alcohol obtenido, encontrándose que en ninguno de los tratamientos se presentó alteración de olor y/o sabor.

Por último, se realizó un análisis comparativo del costo del etanol producido con el sustrato tradicionalmente utilizado en la Destilería (cebada malteada), frente al obtenido utilizando cebada sin maltear adicionada de enzimas a una concentración de $1 \mathrm{gL}^{-1}$, tomando los rendimientos obtenidos en el presente estudio. Se encontró que el costo del etanol producido por hidrólisis enzimática es tres veces más alto que el obtenido por el proceso tradicional, lo que haría inviable el empleo de las enzimas como única herramienta hidrolítica sustituyente de la cebada malteada importada. Sin embargo, en este estudio se demostró que las enzimas empleadas para el proceso amilolítico, realmente hidrolizan el almidón de la cebada sin maltear, resultando en sustratos apropiados para la producción de etanol por $S$. cerevisiae; por lo que se podría considerar para futuros estudios el empleo de concentraciones de sustrato mayores, empleando las mismas concentraciones de enzimas de este estudio, durante periodos de tiempo más prolongados; optimizar otras condiciones en el proceso como el uso de soportes para inmovilización de las enzimas de manera que se puedan reutilizar en el proceso y de esta manera disminuir costos y por último, evaluar mezclas de cebada sin maltear hidrolizada con estas enzimas y de cebada malteada, teniendo en cuenta que entre mayor sea el uso de la cebada nacional o sin maltear en el proceso productivo, mayor será el beneficio para la destilería, no solamente por el costo de esta última sino por los problemas de disponibilidad en algunas épocas.

\section{Conclusiones}

Los resultados obtenidos en el presente estudio, muestran que se puede producir etanol a partir de almidón de cebada sin maltear empleando $\alpha$ y $\beta$-amilasas comerciales. Se obtuvo un porcentaje de hidrólisis del almidón de $89,4 \%$ cuando se adicionaron las enzimas a una concentración de $1 \mathrm{gL}-1$, adicionalmente se obtuvo la máxima producción 
de etanol (5,02\%), siendo significativamente más alta que cuando se utilizó cebada malteada (3,76 \%). Sin embargo, se requiere de más estudios de optimización debido a que los costos de este tratamiento continúan siendo altos en comparación con la utilización de cebada malteada.

\section{Agradecimientos}

El presente trabajo es el resultado del interés de la empresa Destilería Premier LTDA., en especial del Ingeniero Helberth Espitia, Director Técnico de la misma, por su continua búsqueda de alternativas de mejoramiento del proceso a nivel de rendimientos, calidad y rentabilidad. Al Ingeniero Axel, director del laboratorio de Bromatología de la Secretaría de Salud del Tolima de la ciudad de Ibagué, por su asesoría y colaboración en la realización de los análisis.

\section{Conflicto de intereses}

Los autores declaran que no hay conflictos de intereses.

\section{Referencias}

1. Schmitt MR, Marinac L. Beta-amylase degradation by serine endoproteinases from green barley malt, Journal of Cereal Science 2008; 47: 480 - 488.

2. Jones BL. Endoproteases of barley and malt, Journal of Cereal Science 2008; 47: 480 - 488.

3. Departamento Nacional de Planeación. Cerveza, malta y licores. http://www.dnp.gov.co/archivos/documentos/ DDE_Desarrollo_Emp_Industria/Cerveza.pdf. Octubre 11 de 2009

4. Kirk O, Vedel Borchert T, Crone Fuglsang C. Industrial enzyme applications, Current Opinion in Biotechnology 2002; 13: 345 - 351.

5. Cherry JR, Fidantsef AL. Directed evolution of industrial enzymes: an update, Current Opinion in Biotechnology 2003; 14: 438 - 443.

6. Govindasamy S, Campanella OH, Oates CG. Enzymatic hydrolysis and saccharification optimisation of sago starch in a twin-screw extruder, Journal of Food Engineering 1997; 32: 427-446.

7. Kolusheva T, Marinova A. A study of the optimal conditions for starch hydrolysis through thermostable á amylase, Journal of Chemical Technology and Metallurgy 2007; 42 (1): 93-96.
8. Cáceres FM, Lappe P, Larqué SA, Magdub MA, Barahona PL. Ethanol production from henequen (Agave fourcroydes Lem.) juice and molasses by a mixture of two yeasts, Bioresource Technology 2008; 63 (1-4).

9. Frigard T, Andersson R, Arman P. Gradual enzymatic modification of barley and potato amylopectin, Carbohydrate Polymers 2002; 47: 169-179.

10. Linde M, Galbe M, Zacchi G. Bioethanol production from non-starch carbohydrate residues in process streams from a dry-mill ethanol plant, Bioresource Technology 2008; 99: 6505-6511.

11. Shariffa Y, Karim A, Fazilah A, Zaidul I. Enzymatic hydrolysis of granular native and mildly heat-treated tapioca and sweet potato starches at sub-gelatinization temperature, Food Hydrocolloids 2008; 9: 1-7.

12. Ebrahimi F, Khanahmadib M, Roodpeymaa S, J. TM. Ethanol production from bread residues, Biomass and Bioenergy 2007; 7: 1-5.

13. Chen M, Xia L, Xue P. Enzymatic hydrolysis of corncob and ethanol production from cellulosic hydrolysate, International Biodeterior Biodegradation. 2007; 59: $85-89$.

14. Siqueira P, Karp S, Carvalho J, Sturm W, Rodríguez L, Tholozan J, Singhania R, Pandey A, Soccol C. Production of bio-ethanol from soybean molasses by Saccharomyces cerevisiae at laboratory, pilot and industrial scales, Bioresource Technology 2008; 99: 8156-8163.

15. Alfenore S, Molina-Jouve C, Guillouet SE, Uribelarrea JL, Goma G, Benbadis L. Improving ethanol production and viability of Saccharomyces cerevisiae by a vitamin feeding strategy during fed-batch process, Applied Microbiology Biotechnology 2002; 60 (1-2): 67-72.

16. Instituto Nacional De Nomas Técnicas y Certificación (ICONTEC). 2008. Compendio Normas técnicas y guías de implementación de normas del sector bebidas alcohólicas. NTC 5146,

17. Instituto Nacional De Nomas Técnicas y Certificación (ICONTEC). 2008. Compendio Normas técnicas y guías de implementación de normas del sector bebidas alcohólicas. NTC 5113,

18. Schmitt M, Marinac L. Beta-amylase degradation by serine endoproteinases from green barley malt, Journal of Cereal Science 2008; 47: 480-488. 
19. Evans DE, MacLeod LC, Eglinton JK, Gibson CE, Zhang X, Wallace W, Skerritt JH, Lance RCM. Measurement of Beta-amylase in malting barley (Hordeum vulgare L.). I. Development of a quantitative ELISA for Beta-amylase, Journal of Cereal Science 1997; 26:229-239.

20. Fornairon-Bonnefond C, Aguera E, Deytieux C, Sablayrolles J, Salmon J. Impact of Oxygen Addition during Enological Fermentation on Sterol Contents in Yeast Lees and Their Reactivity towards Oxygen,
Journal of Bioscience and Bioengineering 2003; 95 (5): $496-503$.

21. Folch-Mallol JL, Garay-Arroyo A, Lledías F, Covarrubias AA. La respuesta a estrés en la levadura Saccharomyces cerevisiae, Revista Latinoamericana de Microbiología 2004; 46 (1 - 2): 24 - 46.

22. Glazer N. Alexander NH, Microbial Biotechnology. Fundamentals of Applied Microbiology, 2a ed., W.H. Freeman and Company, New York 1998. 